\title{
Electroweak magnetic monopole: The lower mass bound
}

\author{
Petr Beneš ${ }^{a, *}$ and Filip Blaschke ${ }^{a, b}$ \\ ${ }^{a}$ Institute of Experimental and Applied Physics \\ Czech Technical University in Prague \\ Husova 240/5, 11000 Prague 1, Czech Republic \\ ${ }^{b}$ Institute of Physics and Research Centre of Theoretical Physics and Astrophysics \\ Faculty of Philosophy and Science, Silesian University in Opava \\ Bezručovo náměstí 1150/13, 74601 Opava, Czech Republic \\ E-mail: petr.benes@utef.cvut.cz
}

We present exact solutions to the electroweak Cho-Maison magnetic monopole in a family of effective extensions of Standard Model that have a Bogomol'nyi-Prasad-Sommerfield (BPS) limit. We find that the lower bound to the mass of the magnetic monopole is $M \geq 2 \pi v / g \approx 2.37 \mathrm{TeV}$.

We argue that this bound holds universally, not just in theories with a BPS limit.

40th International Conference on High Energy physics - ICHEP2020

July 28 - August 6, 2020

Prague, Czech Republic (virtual meeting)

\footnotetext{
${ }^{*}$ Speaker
} 


\section{Introduction}

Common wisdom is that the Standard Model (SM) doesn't support a magnetic monopole of the 't Hooft-Polyakov type [1,2]. This is of course due to the fact that the second homotopy group of the SM vacuum manifold is trivial: $\pi_{2}\left(S U(2)_{\mathrm{L}} \times U(1)_{\mathrm{Y}} / U(1)_{\mathrm{em}}\right)=\{1\}$.

However, it turns out that the desired topology that could support the solitonic monopole solution can be found elsewhere, namely in the Higgs doublet field $H$ itself, as shown by Cho and Maison [3]. This can be seen as follows. Let us first write the Higgs doublet field as $H=\frac{v}{\sqrt{2}} \rho \xi$, where $\xi$ is a complex doublet normalized as $\xi^{\dagger} \xi=1$ and $v=246 \mathrm{GeV}$ is the vacuum expectation value. Now it can be shown that the field $\xi$ is in fact a $\mathbb{C P}^{1}$ coordinate, due to the $U(1)_{Y}$ symmetry. But its second homotopy group is non-trivial, $\pi_{2}\left(\mathbb{C P}^{1}\right)=\mathbb{Z}$ ! Thus, a stable soliton solutions (having the properties of a magnetic monopole) can, potentially, exist in SM.

Indeed, such a solution can be found. However, a simple calculation reveals that it has an infinite mass: $M=\frac{2 \pi}{g^{\prime 2}} \int_{0}^{\infty} \frac{\mathrm{d} r}{r^{2}}+$ finite terms $=\infty$. Thus, the common wisdom that there is no (physical, finite-mass) magnetic monopole in SM seems justified.

Thus, one must step beyond the SM and modify it in order to get a finite-mass monopole. One way to do that is due to Cho, Kim, and Yoon (CKY) [4] and consists of modifying the hypercharge kinetic term as

$$
-\frac{1}{4 g^{\prime 2}} B_{\mu \nu} B^{\mu \nu} \quad \longrightarrow-\frac{1}{4 g^{\prime 2}} \epsilon\left(|H|^{2}\right) B_{\mu \nu} B^{\mu \nu}
$$

where $\epsilon$ is some positive function of the Higgs field squared, normalized as $\epsilon\left(v^{2} / 2\right)=1$ (in order to recover the SM in the vacuum). Crucially, if $\epsilon \rightarrow 0$ sufficiently fast as $|H|^{2} \rightarrow 0$, the mass of the Cho-Maison monopole comes out finite, as desired. In fact, models beyond SM of this type have indeed been proposed in the literature [5, 6].

Since $\epsilon$ is otherwise virtually arbitrary, also the corresponding monopole mass is in principle arbitrary. E.g., Ellis, Mavromatos and You [7] were varying $\epsilon$ (while keeping consistency with experimental bounds on $H \rightarrow \gamma \gamma$ production) and found a whole variety of monopole masses reaching as low as to $5.5 \mathrm{TeV}$. Thus, a question of phenomenological importance arises whether the monopole can be arbitrarily light or whether there exists some limit.

\section{BPS theory and mass bound}

Indeed, as showed recently, it turns out that there exists a definite lower bound for the monopole mass [8]. In order to derive it, a Bogomol'nyi-Prasad-Sommerfield (BPS) limit of the (bosonic part of the) Standard Model that supports a finite-mass Cho-Maison monopole was constructed:

$$
\begin{aligned}
\mathcal{L}_{\mathrm{BPS}}=\left|D_{\mu} H\right|^{2} & -\frac{v^{2}}{4 g^{2}|H|^{2}} h^{2}\left(\operatorname{Tr}\left[F_{\mu \nu}^{2}\right]-\frac{2}{|H|^{4}} \operatorname{Tr}\left[F_{\mu \nu} H H^{\dagger}\right]^{2}\right) \\
& -\frac{1}{4}\left(\frac{h^{\prime}}{g|H|^{2}} \operatorname{Tr}\left[F_{\mu \nu} H H^{\dagger}\right]+\frac{f^{\prime}}{g^{\prime}} B_{\mu \nu}\right)^{2},
\end{aligned}
$$

where $f^{\prime}(\rho)$ and $h(\rho)$ are arbitrary functions that only have to satisfy the normalization $f^{\prime}(1)=$ $h(1)=1$ and $f^{\prime}(0)=0$. 
Since this is a BPS theory, the equations of motion are, by definition, only of the first order:

$$
D_{i} H=\frac{\sqrt{2}}{g \rho} h(\rho)\left(M_{i}-\xi^{\dagger} M_{i} \xi\right) \xi+\frac{1}{\sqrt{2} g} h^{\prime}(\rho)\left(\xi^{\dagger} M_{i} \xi\right) \xi+\frac{1}{\sqrt{2} g^{\prime}} f^{\prime}(\rho) G_{i} \xi,
$$

where $M_{i}=\frac{1}{2} \varepsilon_{i j k} F_{j k}^{a} \sigma_{a}$ and $G_{i}=\frac{1}{2} \varepsilon_{i j k} B_{j k}$. Consequently, they are easier to solve. Indeed, for for some choices of $f$ and $h$ exact solutions, describing a single spherically-symmetric monopole, can be found.

More important is that any solution of the BPS theory satisfies the energetic Bogomol'nyi bound, that is, the mass of a given static solution is the lowest possible. In particular, mass of a static spherically-symmetric monopole solution can be found, without having to solve the equations of motion at all, to be

$$
M=4 \pi v\left[\frac{1}{2 g}+\frac{1}{g^{\prime}}\left|\int_{0}^{1} \mathrm{~d} \rho f^{\prime}(\rho)\right|\right] .
$$

From this the monopole lower mass bound follows immediately:

$$
M \geq \frac{2 \pi v}{g} \approx 2.37 \mathrm{TeV}
$$

This bound can be shown to hold not only in a BPS theory, but also in a more realistic theory with the CKY regularization of the monopole mass. Thus, we conclude that it is in principle possible, at least kinematically, that a magnetic monopole could be pair-produced at the LHC and discovered in the dedicated MoEDAL experiment [9].

\section{Acknowledgments}

This work was supported by the Albert Einstein Centre for Gravitation and Astrophysics financed by the Czech Science Agency Grant No. 14-37086G (F. B.) and by the program of Czech Ministry of Education, Youth and Sports INTEREXCELLENCE Grant No. LTT17018 (F. B., P. B.). P. B. also thanks TJ Balvan Praha for support.

\section{References}

[1] 't Hooft G 1974 Nucl. Phys. B79 276-284

[2] Polyakov A M 1974 JETP Lett. 20 194-195 [Pisma Zh. Eksp. Teor. Fiz.20,430(1974)]

[3] Cho Y M and Maison D 1997 Phys. Lett. B391 360-365 (Preprint hep-th/9601028)

[4] Cho Y M, Kim K and Yoon J H 2015 Eur. Phys. J. C75 67 (Preprint 1305 . 1699)

[5] Arai M, Blaschke F, Eto M and Sakai N 2017 (Preprint 1703.00351)

[6] Arai M, Blaschke F, Eto M and Sakai N 2017 PTEP 2017 053B01 (Preprint 1703. 00427)

[7] Ellis J, Mavromatos N E and You T 2016 Phys. Lett. B756 29-35 (Preprint 1602 . 01745)

[8] Blaschke F and Beneš P 2018 PTEP $2018073 B 03$ (Preprint 1711. 04842)

[9] Acharya B et al. (MoEDAL) 2014 Int. J. Mod. Phys. A 291430050 (Preprint 1405. 7662) 